\title{
Morphometric Analysis of 3D Soft-Tissue for Sexual Dimorphism in Human Face
}

\author{
Análisis Morfométrico de Tejidos Blandos 3D de Dimorfismo Sexual en Rostro Humano
}

\author{
Olalekan Agbolade ${ }^{1,2}$; Azree Nazri ${ }^{1,2}$; Razali Yaakob ${ }^{1}$; Abdul Azim Ghani ${ }^{3}$ \& Yoke Kqueen Cheah $^{4}$
}

AGBOlade, O.; NAZRI, A.; YAAKOB, R.; GHANI, A. A. \& CHEAH, Y. K. Morphometric analysis of 3D soft-tissue for sexual dimorphism in human face. Int. J Morphol., 38(2):367-373, 2020.

SUMMARY: Sexual dimorphism in Homo-sapiens is a phenomenon of a direct product of evolution by natural selection where evolutionary forces acted separately on the sexes which brought about the differences in appearance between male and female such as in shape and size. Advances in morphometrics have skyrocketed the rate of research on sex differences in human and other species. However, the current challenges facing 3D in the acquisition of facial data such as lack of homology, insufficient landmarks to characterize the facial shape and complex computational process for facial point digitization require further study in the domain of sex dimorphism. This study investigates sexual dimorphism in the human face with the application of Automatic Homologous Multi-points Warping (AHMW) for 3D facial landmark by building a template mesh as a reference object which is thereby applied to each of the target mesh on Stirling/ESRC dataset containing 101 subjects ( $m a l e=47$, female $=54$ ). The semi-landmarks are subjected to sliding along tangents to the curves and surfaces until the bending energy between a template and a target form is minimal. Principal Component Analysis (PCA) is used for feature selection and the features are classified using Linear Discriminant Analysis (LDA) with an accuracy of 99.01 $\%$ which demonstrates that the method is robust.

KEY WORDS: Sexual dimorphism; Facial landmark; 3D geometric morphometrics; Multi-point warping; LDA.

\section{INTRODUCTION}

Identification of sexes plays a remarkable role when it comes to social communication. This identification of sexes by human beings is relatively easy and accurate. However, achieving the same result with classification through machine remains a challenge in computer vision. As the face is the part that hosts the most crucial sensory organs and acts as the central interface for appearance, communication, expression, and identification (Peng et al., 2013).

Morphometric examines shape variation, group differences in shape, central tendency of shape, and associations of shape with extrinsic factors (Slice, 2007). In morphometry, sliding semi-landmarks have been used in the study of bone surface such as articular and the diaphysis (Fabre et al., 2014) and curves, providing descriptors of outlines and crests (De Groote et al., 2010;
Alvarez et al., 2013). Sliding semi-landmark was used in (Perez et al., 2006) to investigate craniofacial and dental variation in human, by minimizing bending energy and Procrustes distance. Sliding semi-landmarks are used in the study of surfaces and curves on meshes. To circumvent the problem of asymmetry caused by manual semilandmarks, sliding semi-landmarks that are relaxed against a symmetrized mean using bending energy minimization was proposed in (Schlager \& Rüdell, 2015), in the investigation of nasal soft tissue reconstruction. To evaluate difference software packages for semi-landmark, BottonDivet et al. (2015) used sliding semi-landmark to analyze the workflow complexity and time consumption to complete the sliding task. However, analyzing facial variation in soft-tissue for sexual dimorphism in human from sliding semi-landmark is not prevalent in the three dimensional model.

\footnotetext{
${ }^{1}$ Department of Computer Science, Faculty of Computer Science \& IT, Universiti Putra Malaysia, Selangor, Malaysia.

${ }^{2}$ Institute of Bioscience, Universiti Putra Malaysia, Selangor, Malaysia.

${ }^{3}$ Department of Software Engineering, Faculty of Computer Science \& IT, Universiti Putra Malaysia, Selangor, Malaysia.

${ }^{4}$ Department of Biomedical Science, Faculty of Medicine and Health Sciences, Universiti Putra Malaysia, Selangor, Malaysia.

FUNDING: This work was supported by Fundamental Research Grant Scheme, Ministry of Higher Education, Malaysia (MOHE)-FRGS Code: 5524959. And Putra German UPM-Code:9538100.
} 
This work aims to investigate whether geometric morphometric analyses of soft-tissue landmarks using multipoint warping is reliable to assess sex differences in the human face. This is done by projecting the surface semilandmarks from the template object to the target objects and iteratively slides the semi-landmarks to a point relaxed. Here we used six iterations to ensure convergence and optimum smoothness. This method is not new, in analyzing shape variation in geometry morphometric, but its application to the analysis of shape variation for soft-tissue three-dimensional sexual dimorphism in human face is novel and the simplicity of the workflow requires to performing the semilandmark sliding task in Viewbox 4.0. The results are further used to investigate size and shape variation in Stirling dataset to identify the features that are most dimorphic in male and female faces; as the features responsible for dimorphism in humans are still under study (Samal et al., 2007).

\section{MATERIAL AND METHOD}

Dataset and description. The dataset is acquired from Stirling/ESRC 3D Face Database captured by a Di3D camera system (Stirling-ESRC, 2018). The image format is in wavefront obj file containing 101 subjects (male $=47$, female $=54$ ) of $3 \mathrm{D}$ facial scans in neutral expression were randomly selected which are intended to facilitate research in sexual dimorphism, face recognition, expression recognition, and perception. The dataset is being used as a test set for a competition on $3 \mathrm{D}$ face reconstruction from $2 \mathrm{D}$ images, with the 3D scans acting as 'ground truth' in IEEE conference.

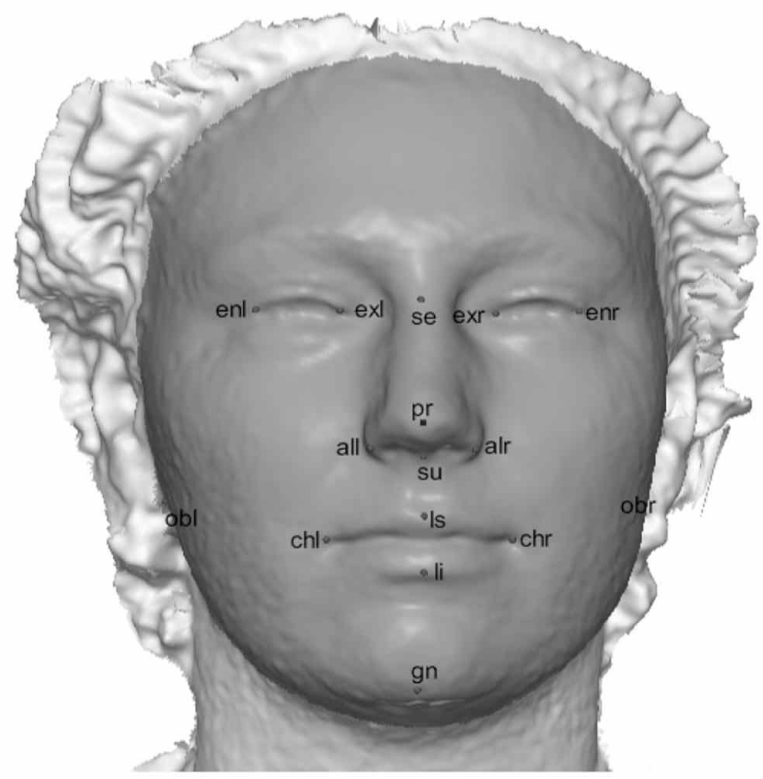

Fig. 1 A three-dimensional mesh template showing 16 fixed anatomical landmarks.
Multi-Point Warping Approach. The template mesh is created by manually locating sixteen anatomical points on the 3D face (Fig. 1) called anchor points according to 3D facial landmark standard in (Caple \& Stephan, 2016) with little modification (details in Table I).

The anchor landmarks are not subjected to sliding, but are used for establishing the warping fields that will be used for minimizing the bending energy. Due to the easy detection, pose correction and invariance to facial expression of nose tip, the nose tip (pronasale) was selected as the most robust and prominent landmark point. Since the nose tip area can be approximated as a semi-sphere of the human face. This is where the sliding points begin to spread across the facial surface. Using this anchor point (pronasale), 484 semilandmarks were automatically generated overlapping on each other at the pronasale region showing in blue color. These are uniformly and randomly distributed on the selected facial surface with $1.5 \mathrm{~mm}$ radius to accommodate all 500 points using method in (Zelditch et al., 2012). And the landmarks sliding and acquisition is implemented in Viewbox 4.0 software (Halazonetis, 2018).

The semi-landmarks are allowed to slide on the curve and surface mesh of each target using TPS warping of the template. This positions the reference points on the target facial mesh by minimizing the bending energy. Because warping may result in points that do not lie directly on the facial surface on the target mesh (Figs. 2A,B), the transferred points are projected on the closest point on the mesh surface using ICP method (Creusot et al., 2010) which aims to iteratively minimize the mean square error between two point sets. If the distance between the two points is within an acceptable threshold, then the closest point is determined as the corresponding point. During the relaxation of the spline, the semi-landmarks slide along the surface and the curve tangent structures and not on the surfaces or the curves which reduces the computational effort, as the minimization problem became linear. This is because the sliding along the tangents lets the semi landmarks slip off the data and the target surface mesh is then considered homologous (Figs. 2C, D).

In assessing error, six subjects (three males and three females) from the sample are randomly selected; each one belonging to a different individual, distinct from the template subject. Each was digitized twice following the same method to account for digitization error. The results are analyzed using Procrustes ANOVA. This is done by the minimization of the squared sum of the distance of all objects and the consensus configuration (Fruciano, 2016).

PCA and LDA. Due to a large number of facial landmarks, landmark coordinates were decomposed into 
Table I. Anchor anatomical points and descriptions.

\begin{tabular}{|c|c|c|c|}
\hline No & Anchor Landmarks & 3D Notation & Description \\
\hline & Eyes Region & & \\
\hline 1 & Endocanthion left & Enl & $\begin{array}{l}\text { Left most medial point of the palpebral fissure, at the inner commissure } \\
\text { of the eye }\end{array}$ \\
\hline 2 & Exocanthion left & Exl & $\begin{array}{l}\text { Left most lateral point of the palpebral fissure, at the outer commissure } \\
\text { of the eye }\end{array}$ \\
\hline 3 & Exocanthion right & Exr & $\begin{array}{l}\text { Right most lateral point of the palpebral fissure, at the outer } \\
\text { commissure of the eye }\end{array}$ \\
\hline 4 & Endocanthion right & enr & $\begin{array}{l}\text { Right most medial point of the palpebral fissure, at the inner } \\
\text { commissure of the eye }\end{array}$ \\
\hline \multirow[t]{2}{*}{5} & Sellion & se & Deepest midline point of the nasofrontal angle \\
\hline & Nasal Region & & \\
\hline 6 & Pronasale & $\mathrm{pr}$ & The most anteriorly protruded point of the apex nasi \\
\hline 7 & subnasale & su & $\begin{array}{l}\text { Median point at the junction between the lower margin of the nasal } \\
\text { septum and the philtrum area }\end{array}$ \\
\hline 8 & Alare left & all & Left most lateral point on the nasal ala \\
\hline \multirow[t]{2}{*}{9} & Alare right & alr & Right most lateral point on the nasal ala \\
\hline & Mouth Region & & \\
\hline 10 & Cheilion lef $\mathrm{t}$ & chl & $\begin{array}{l}\text { Left outer corners of the mouth where the outer edges of the upper and } \\
\text { lower vermilions meet }\end{array}$ \\
\hline 11 & Cheilion right & $\operatorname{chr}$ & $\begin{array}{l}\text { Right outer corners of the mouth where the outer edges of the upper } \\
\text { and lower vermilions meet }\end{array}$ \\
\hline 12 & Labiale superius & 1s & Midpoint of the vermilion margin of the upper lip \\
\hline 13 & Labiale inferius & li & Midpoint of the vermilion margin of the lower lip \\
\hline 14 & $\begin{array}{l}\text { Chin-Cheek Region } \\
\text { Gnathion }\end{array}$ & gn & $\begin{array}{l}\text { Median point halfway between pogonion and menton } \\
\text { Left median point where the sagittal suture intersec ts with a transverse }\end{array}$ \\
\hline 15 & Obelion left & obl & line connecting parietal foramina \\
\hline 16 & Obelion right & obr & $\begin{array}{l}\text { Right median point where the sagittal suture intersects with a } \\
\text { transverse line connecting parietal foramina }\end{array}$ \\
\hline
\end{tabular}

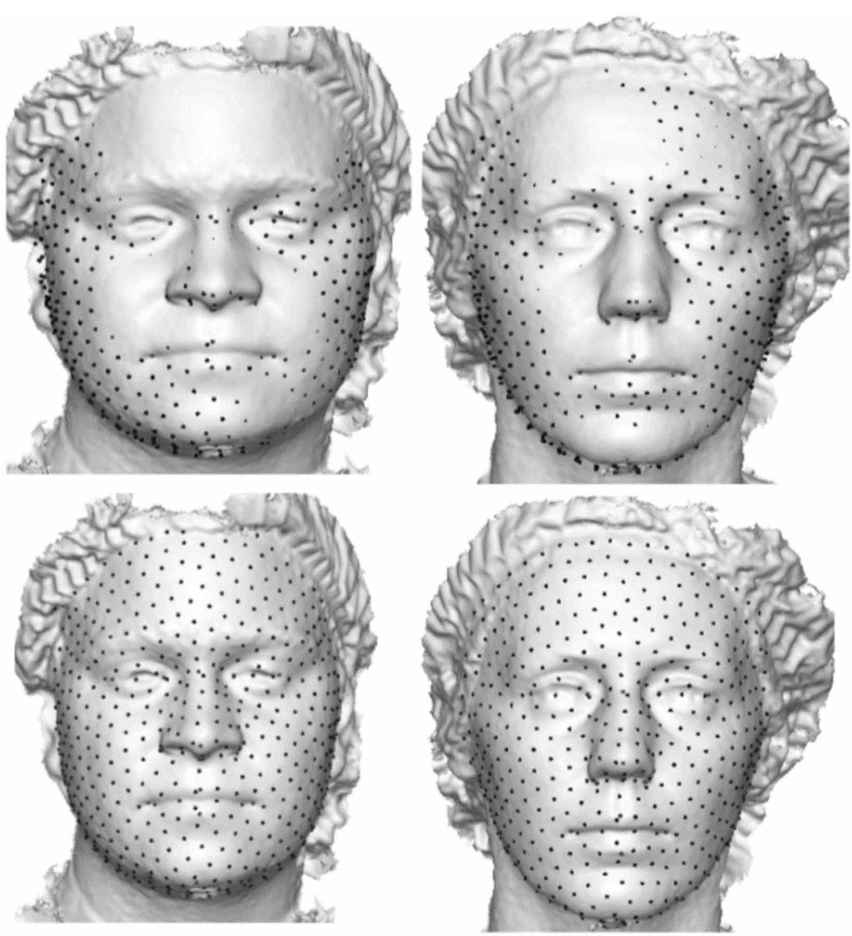

their principal components and computed to account for the proportion of variation and its significance. The first seven principal components accounting for over $80 \%$ of the variation and the PCAs are used to characterize the features of shape variation. To compare sizes, CS (Centroid Size) between sexes, values were logtransformed and Mann-Whitney U test was used for significant differences in $\log$ CS medians between sexes and the Kolmogorov-Smirnov D tests for overall equal distribution of both sexes as CS does not assume a normal distribution. To correct the effect of size on the facial shape (Klingenberg \& McIntyre, 1998), allometry effect is explored to compute multivariate regression of shape on log centroid size in MorphoJ. Subsequently, differences in effects and size are examined by computing non-parametric analysis of variance

Fig. 2 Sliding point warped on target facial surface. (A) Male partial sliding on target mesh. (B) Female partial sliding on target mesh. (C) Male complete and homologous warping on target mesh. (D) Female complete and homologous warping on target mesh. 
(MANOVA) in terms of Wilks' lambda in PAST software. Using sex as a group and size as the covariate, the sex by size interaction term is performed. The MANOVA is recomputed after removing the interaction term (sex by size) and the sex effect tests difference in regression intercept. The significant level for all tests is accepted at $p<0.05$. To predict the classification accuracy of the sexes, we apply LDA in PAST software.

\section{RESULTS}

Significance of Landmark. The overall landmarks are tested using ANOVA to see the significance of the variation on the overall landmarks in each sex group. Male: $\mathrm{F}=3045$, df $=$ 1391, p-value $=0.00001 ;$ Female: $\mathrm{F}=3638, \mathrm{df}=1391, \mathrm{p}-$ value $=0.00001$. Furthermore, we conduct PERMANOVA (Non-Parametric MANOVA) which is a non-parametric test of the significant difference between the sex groups based on the distance measured (Anderson, 2001) with $\mathrm{F}=17.33$, $p=0.00001$ and $r=0.98$. The large positive of $F$ value indicates that there is a significant difference between the sex groups.

Error Assessment, Size and Shape Variation. For the assessment of digitization errors of the overall landmarks; the deviations of each landmark is obtained by simply

Table II. Procrustes ANOVAs for facial shape

\begin{tabular}{lccccc}
\hline \multicolumn{1}{c}{ Effect } & SS & MS & DF & F & P \\
\hline Sex & 0.011884 & $7.96 \mathrm{E}-06$ & 1493 & 3.22 & $<.0001$ \\
Individual & 0.206839 & $2.47 \mathrm{E}-06$ & 83608 & 0.89 & \\
Error & 0.02495 & $2.79 \mathrm{E}-06$ & 8958 & & \\
Total & 0.243673 & $1.32 \mathrm{E}-05$ & 94059 & & \\
\hline
\end{tabular}

SS: sum of squares; MS: mean square; DF: degrees of freedom; F: F-statistic; P: $\mathrm{P}$-value (parametric) calculating the amount of displacement from the average position calculated from all digitization and the variation accounts for the smallest portion of the total variation using Procrustes ANOVA. The digitization error accounts for only 0.025 from the total variation (Table II).

To visualize the shape changes with landmark displacement after Procrustes superimposition, a lollipop graph is plotted where the landmark positions of the starting shape are denoted by dots (candy) and the shifts of the landmark to the target shape are denoted as lines (stick) (Klingenberg, 2013). This visualization is sometimes cumbersome in a three-dimensional context which has been applied in many morphometrics studies such as in (Rohlf, 1993). Because lollipop graph provides little information underlying anatomical structure, more sophisticated relative warped graphs are plotted after Procrustes fit to provide more information of the position of shape changes using PCs as a $3 \mathrm{D}$ vector from the mean configuration (Dryden, 2014).

The first 7 PCs of the PCA explain more than $80 \%$ of the variance; $\mathrm{PC} 1$ explains 37.25 of the variance followed by PC2 with $11.91 \%$ variance. The log centroid size for multivariate regression is only slightly different between the sexes $(\mathrm{U}=1134, \mathrm{p}=0.25)$ (Fig. 3A), therefore not significant, though the test for equal distribution is significant $(\mathrm{D}=2.08$, $\mathrm{p}=0.024)$. Inter-individual allometry explains only $2.65 \%$ of shape differences according to size (Fig. 3B) and therefore is not significant $(\mathrm{p}=0.11)$. The regression results indicate that there is some weak evidence against the null hypothesis of independence. Given the sample size $(\mathrm{N}=101)$ and the high dimensionality data; it is also to be expected that the power of the test is low (Klingenberg et al., 2002). The readers should, therefore, interpret the regression results with caution.

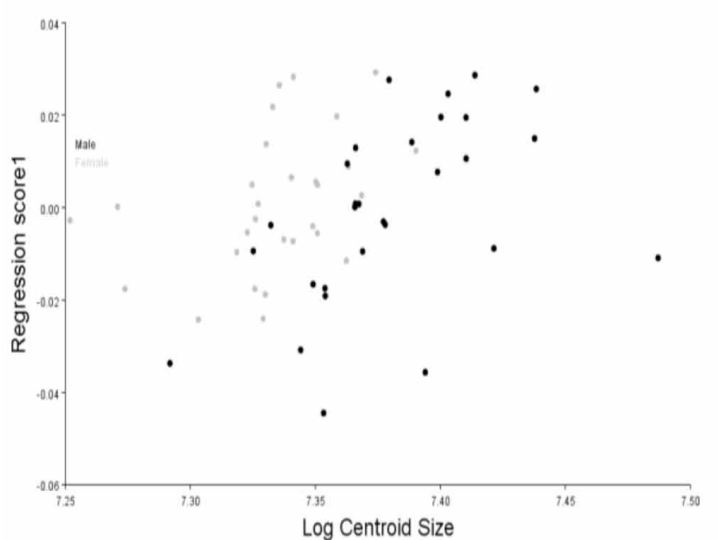

A

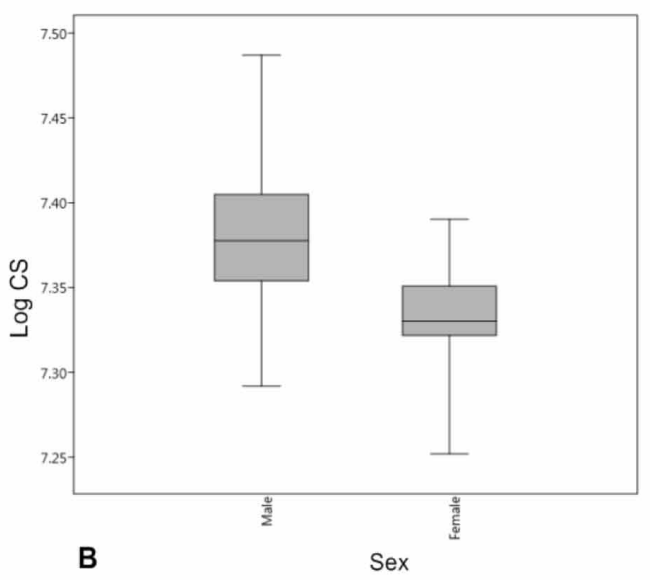

Sex

Fig. 3. Multivariate regression and boxplot of the shape variables on log centroid size. (A) Male is represented by blue color and female is represented by red color. (B) Boxplot for centroid size for males and female. 


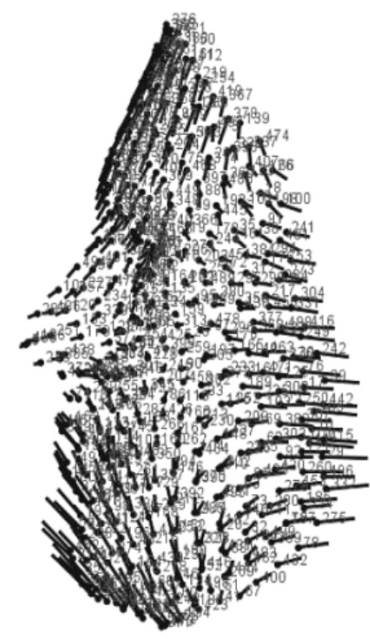

A

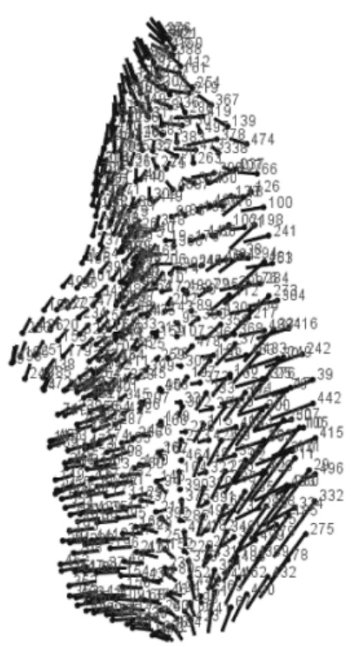

B

Fig. 4. Lollipop Graphs visualizing face shape changes. A (male), B (female).

The interaction term (test for slopes) is statistically significant (Table III). When the size effect is removed and the MANOVA is repeated, the result is still statistically significant. This suggests that the effect of size on shape for both slope and intercept is strong and not similar in the sex group.

Table III. MANOVA results in terms of Wilks' Lambda.

\begin{tabular}{cccccc}
\hline Effect & Wilk & df1 & df2 & f & P-value \\
\hline Sex x CS & 0.301 & 8 & 91 & 26.42 & $<0.000$ \\
Sex & 0.3015 & 7 & 92 & 30.44 & $<0.000$ \\
\hline
\end{tabular}
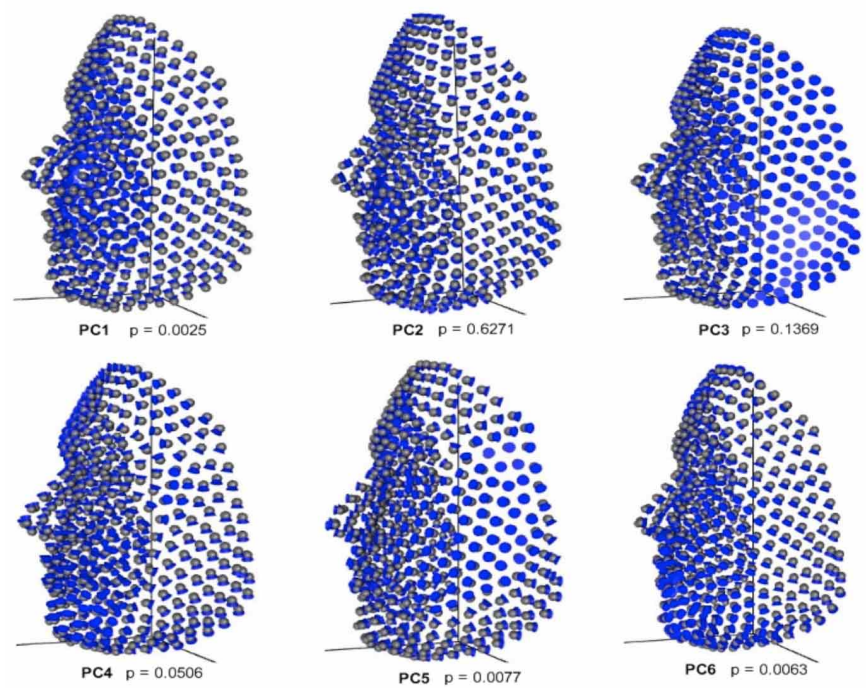

Table IV. Confusion matrix for actual and predicted sex

\begin{tabular}{cccc}
\hline Actual/Predicted & Female & Male & Total \\
\hline Female & 51 & 0 & 51 \\
Male & 1 & 49 & 50 \\
Total & 52 & 49 & 101 \\
\hline
\end{tabular}

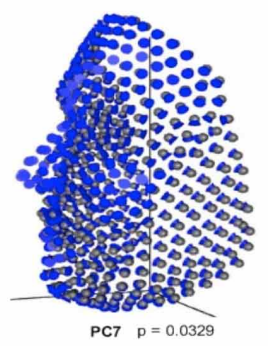

Fig. 5. Relative warps of selected PCs showing dimorphic region in the face with their P-values $(\mathrm{P}<0.05)$ 


\section{DISCUSSION}

The approach in this algorithm uses sliding semilandmark, starting at the pronasale with respect to the arbitrary template model where the bending energy between all subjects is minimized by six cycles iterative sliding. This is important because manual semi-landmarks are not appropriate for the comparison of forms and shapes when the curves and surfaces are not homologous among the targets. Because the point homology across specimens experienced by morphometrics measurement of semi-landmarks on curves and surfaces manually is no longer guaranteed due to the biological meaninglessness and un-interpretable of sample averages and variances (Mitteroecker et al., 2013).

The Procrustes ANOVA suggests a modest but appreciable variation in facial shape. Shape differences are statistically significant even after averaging faces within sex. Small measurement error shows that the landmarks can be annotated with precision using the proposed method. Though, many approaches are available in addressing measurement error. Discussing such at length is beyond the scope of this study, more and extended details can be found in Fruciano.

Allometry in shape is tested by examining the statistical correlation between size and shape. This characterizes the expected shape changes per (centroid size) unit increase size. The statistical significance of the association between shape and size is tested statistically based on Goodall (1991) F statistic. When the factors other than size, have effects on shape variation such as sexual dimorphism, the plots are not optimal. To avoid this, a computation on regression score is performed by projecting data points in shape space unto the axis in the direction of regression vector (Drake \& Klingenberg, 2008). The tests for intercept and slope using MANOVA are both statistically significant. This suggests that the effect of size on shape is strong and not similar in the sex group. As it is expected since sex has a large phenotypic variation, the allometric trajectories are largely aligned with the vector of mean shape differences.

Morphological differences associated with the principal components are linked to sexual dimorphism to explain more anatomical details. Only PC1 $(\mathrm{p}=0.0025)$, PC5 ( $\mathrm{p}=$ $0.0077)$, PC6 $(p=0.0063)$ and PC7 $(p=0.0329)$ are significant; whereas PC2 (p = 0.6271), PC3 (p = 0.1369) and PC4 $(p=0.0506)$ are not significant. The PCs also reveal sexual dimorphic regions: $\mathrm{PC} 1$ and $\mathrm{PC} 2$ (mouth region), $\mathrm{PC} 3$ and PC5 (cheek region), PC4 and PC6 (chin region) and PC7 (nasal region and upper-head region), though upperhead region is not considered in this study.
The female generally has elongated chin height (lign) than the male group, though male chin height is more curved than that of the female. The nasal width (all-alr) is wider in the female than that of male, though mere looking at one face may confuse the viewer, but the average data gathered justifies the reports. Cheek length (gn-obr) in male is longer and more curved than that of the female, though that of female steep downward than that of the male. The nasal bridge length (se-pr) in male is a little longer than that of the female. The female nasal tip (pr-su) is a little bit more protuberant than that of the male but wider in male group. Biocular width (enl-enr) is wider in male than that of the female and upper lip height (su-ls) is longer in female. Morphological characteristics of male and female faces vary in different races or datasets, therefore presenting a consensus may not be scientifically acceptable.

Generally, the male face shape is bigger than the female face shape. We further predict the sexes by employing one of the supervised learning techniques, LDA; and the classifier classified the sex groups with an accuracy of 99.01 $\%$. Though, one male was misclassified as female by the classifier. To the best of our knowledge, there is currently no facial landmark annotation analysis or sexual dimorphism performed using Stirling/ESRC dataset.

\section{CONCLUSIONS}

This method combines pragmatic solutions to configure an optimized pipeline for high-throughput homologous multi-points facial signature in three dimensional to the application of sexual dimorphism. The landmarks accuracy is measured using deviation from the reference surface tothe target surface with Procrustes distance after superimposition and the error rate through Procrustes ANOVA is minimal. The dimorphic regions are identified and visualized using PCA. Though regression results indicate weak evidence of allometry yet the tests for slope and intercept for the effect of size on shape are significant. Such a high-throughput phenotypic facial data with good classification accuracy like this is not only valuable for sexual dimorphism but also in forensic studies of human facial morphology, anthropology, disease diagnosis and prediction, statistical shape or image analysis, face recognition, age estimation, facial expression recognition, etc. This study is based on Stirling/ERSC dataset which is the European population, therefore the methods and results presented here should be tested in other populations. Finally, implementation with deep learning may yield better performance and robust result in the feature with respect to ethnicity and moderate changes in facial features. 


\section{ACKNOWLEDGMENTS}

We acknowledge Stirling/ESRC (University of Stirling) for prompt agreement to use their datasets. Furthermore, credit goes to Computer Laboratory of the Faculty of Computer Science \& Information Technology, Universiti Putra Malaysia.

AGBOLADE, O.; NAZRI, A.; YAAKOB, R.; GHANI, A. A. \& CHEAH, Y. K. Análisis morfométrico de tejidos blandos 3D de dimorfismo sexual en rostro humano. Int. J. Morphol., 38(2):367373,2020 .

RESUMEN: El dimorfismo sexual en el Homo-sapiens es un fenómeno directo de la evolución por selección natural, donde las fuerzas evolutivas actuaron por separado en los sexos, lo que provocó las diferencias en la apariencia entre hombres y mujeres, tal como la forma y tamaño. Los avances en el área de la morfometría, han generado un aumento significativo de las investigaciones en las diferencias de sexo en humanos y otras especies. Sin embargo, los desafíos actuales que enfrenta el 3D en el análisis de datos faciales, como la falta de homología, puntos de referencia insuficientes para caracterizar la forma facial y la complejidad del proceso computacional para la digitalización de puntos faciales, requiere un estudio adicional en el área del dimorfismo sexual. Este estudio investiga el dimorfismo sexual en el rostro humano con la aplicación de la deformación automática de múltiples puntos homólogos para el hito facial 3D, mediante la elaboración de una malla de plantilla como objeto de referencia, y se aplica en cada una de las mallas objetivas en el conjunto de datos Stirling / ESRC que contiene 101 sujetos (hombre $=47$, mujer $=54$ ). Los semi-puntos de referencia se deslizan a lo largo de las tangentes a las curvas y superficies hasta que la energía de flexión entre una plantilla y una forma objetivo es mínima. El análisis de componentes principales (PCA) se utiliza para la selección de características y las características se clasifican mediante el análisis discriminante lineal (ADL) con una precisión del 99,01\%, lo que demuestra la validez del método.

PALABRAS CLAVE: Dimorfismo sexual; Punto de referencia facial; Morfometría geométrica 3D; Deformación multipunto; LDA.

\section{REFERENCES}

Alvarez, A.; Ercoli, M. D. \& Prevosti, F. J. locomotion in some small to mediumsized mammals: a geometric morphometric analysis of the penultimate lumbar vertebra, pelvis and hindlimbs. Zoology (Jena), 116(6):356-71, 2013.

Anderson, M. J. A new method for non-parametric multivariate analysis of variance. Austral Ecol., 26(1):32-46, 2001.

Botton-Divet, L.; Houssaye, A.; Herrel, A.; Fabre, A. C. \& Cornette, R. Tools for quantitative form description; an evaluation of different software packages for semi-landmark analysis. PeerJ, 3:e1417, 2015.

Caple, J. \& Stephan, C. N. A standardized nomenclature for craniofacial and facial anthropometry. Int. J. Legal Med., 130(3):863-79, 2016.
Creusot, C.; Pears, N. \& Austin, J. 3D Face Landmark Labelling. Proceedings of the ACM Workshop on 3D Object Retrieval, 2010.

De Groote, I.; Lockwood, C. A. \& Aiello, L. C. Technical note: A new method for measuring long bone curvature using 3D landmarks and semi-landmarks. Am. J. Phys. Anthropol., 141(4):658-64, 2010.

Drake, A. G. \& Klingenberg, C. P. The pace of morphological change: historical transformation of skull shape in St Bernard dogs. Proc. Biol. Sci., 275(1630):71-6, 2008.

Dryden, I. L. Shape Analysis. Wiley StatsRef: Statistics Reference Online, 2014. doi: 10.1002/9781118445112.stat05087

Fabre, A. C.; Goswami, A.; Peigné, S. \& Cornette, R. Morphological integration in the forelimb of musteloid carnivorans. J. Anat., 225(1):19-30, 2014.

Fruciano, C. Measurement error in geometric morphometrics. Dev. Genes Evol., 226(3):139-58, 2016.

Goodall, C. Procrustes methods in the statistical analysis of shape. J. R. Stat. Soc. Series B Methodol., 53(2):285-321, 1991.

Halazonetis, D. Viewbox 4 - Cephalometric Software. 2018. Available from: http:/ /dhal.com/viewboxindex.htm

Klingenberg, C. P. \& McIntyre, G. S. Geometric morphometrics of developmental instability: Analyzing patterns of fluctuating asymmetry with procrustes methods. Evolution, 52(5):1363-75, 1998.

Klingenberg, C. P. Visualizations in geometric morphometrics: how to read and how to make graphs showing shape changes. Hystrix Ital. J. Mammal., 24(1):15-24, 2013.

Klingenberg, C. P.; Barluenga, M. \& Meyer, A. Shape analysis of symmetric structures: Quantifying variation among individuals and asymmetry. Evolution, 56(10):1909-20, 2002.

Mitteroecker, P.; Gunz, P.; Windhager, S. \& Schaefer, K. A brief review of shape, form, and allometry in geometric morphometrics, with applications to human facial morphology. Hystrix Ital. J. Mammal., 24(1):59-66, 2013.

Nacua, S. S.; Torres, M. A. J. \& Demayo, C. G. Landmark-based geometric morphometrics in visualizing body shape dimorphism in the endemic cyprinid, Puntius tumba (Herre, 1924), from Lake Lanao, Philippines. 2010 International Conference on Environmental Engineering and Applications (ICEEA), 2010.

Peng, S.; Tan, J.; Hu, S.; Zhou, H.; Guo, J.; Jin, L. \& Tang, K. Detecting genetic association of common human facial morphological variation using high density 3D image registration. PLoS Comput. Biol., 9(12):e1003375, 2013.

Perez, S. I.; Bernal, V. \& Gonzalez, P. N. Differences between sliding semilandmark methods in geometric morphometrics, with an application to human craniofacial and dental variation. J. Anat., 208(6):769-84, 2006.

Rohlf, F. J. Relative Warp Analysis and an Example of its Application to Mosquito Wings. New York, State University of New York, 1993.

Samal, A.; Subramani, V. \& Marx, D. Analysis of sexual dimorphism in human face. JoJ. Vis. Commun. Image Represent., 18(6):453-63, 2007.

Schlager, S. \& Rüdell, A. Analysis of the human osseous nasal shape--population differences and sexual dimorphism. Am. J. Phys. Anthropol., 157(4):571-81, 2015.

Slice, D. E. Geometric morphometrics. Ann. Rev. Anthropol., 36(1):261-81, 2007.

Stirling-ESRC. Stirling-ESRC 3D Face Database, 2018. Available from: http:// pics.stir.ac.uk/ESRC/3d_images.htm

Zelditch, M. L.; Swiderski, D. L. \& Sheets, H. D. Geometric Morphometrics for Biologists: A Primer. Amsterdam, Elsevier Academic Press, 2012.

\section{Corresponding author: \\ Dr. Azree Nazri}

Department of Computer Science

Faculty of Computer Science \& IT

Universiti Putra Malaysia

Selangor - MALAYSIA

Email: azree@upm.edu.my

Received: 29-08-2019

Accepted: $14-10-2019$ 